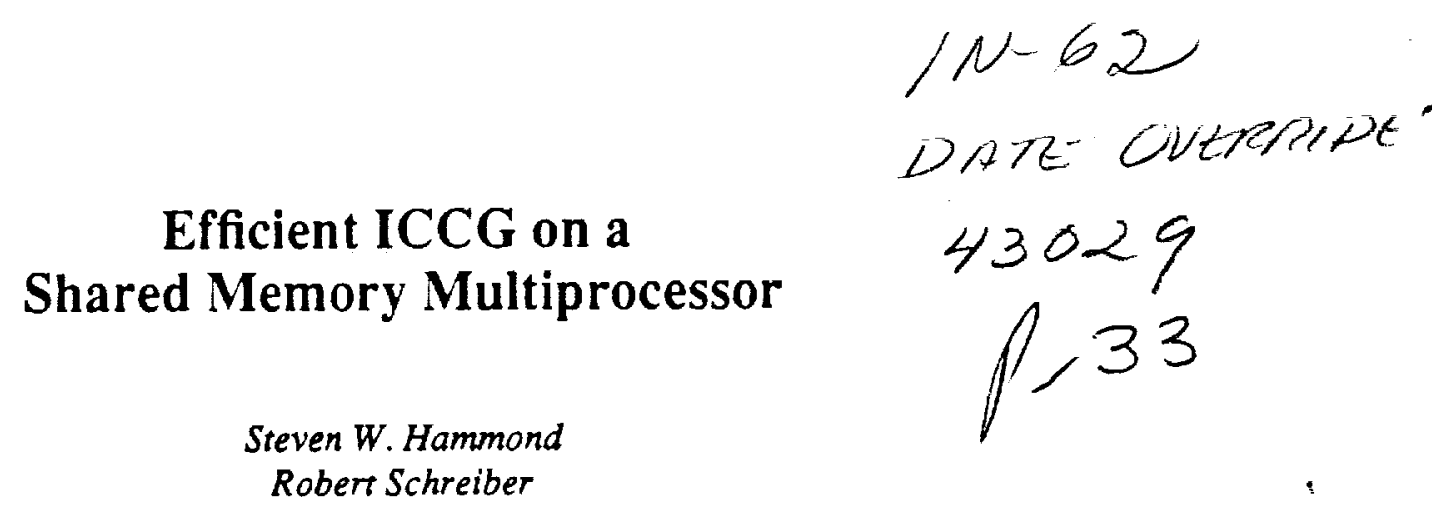

May, 1989

Research Institute for Advanced Computer Science NASA Ames Research Center

RLACS Technical Report 89.24

NASA Cooperative Agreement Number NCC 2-387

(NASA-CR-188843) EFFICIENT ICCG ON A SHARED

N92-11693 MEMORY MULTIPROCESSOR (RESEArCh Inst. FOr Advanced Computer SCience) $33 \mathrm{p}$ CSCL $09 \mathrm{~B}$

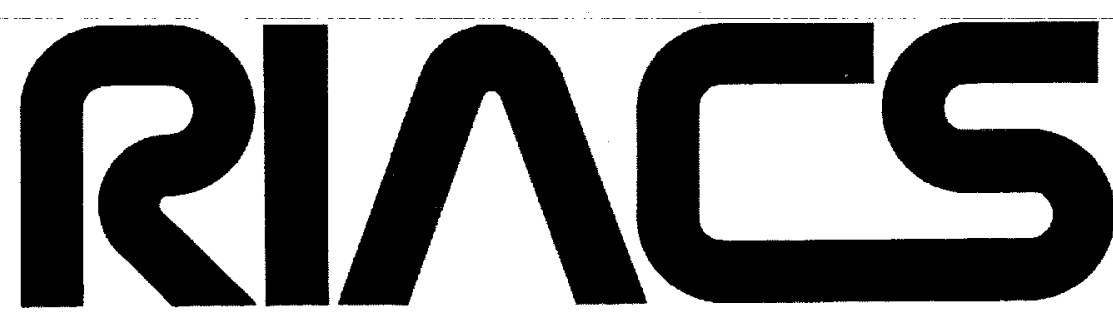

Research Instiłute for Advanced Computer Science 


\title{
Efficient ICCG on a Shared Memory Multiprocessor
}

\author{
Steven W. Hammond \\ Robert Schreiber
}

May, 1989

Research Institute for Advanced Computer Science NASA Ames Research Center

RIACS Technical Report 89.24

NASA Cooperative Agreement Number NCC 2-387 


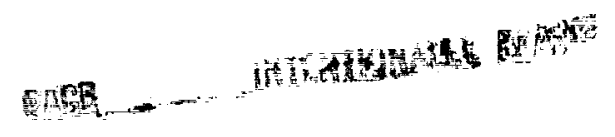




\title{
Efficient ICCG on a Shared Memory Multiprocessor *
}

\author{
Steven W. Hammond ${ }^{\dagger} \quad$ Robert Schreiber ${ }^{\ddagger}$
}

May 1989

\begin{abstract}
In this paper we discuss different approaches for exploiting parallelism in the ICCCi method for solving large sparse symmetric positive lefinite systems of equations on a shared memory parallel computer. Techniques for efficiently solving triangular systems and computing sparse matrix-vector products are explored. Three methods for scheduling the tasks in solving triangılar sy'stems are implemented on the Sequent Balance 21000. Sample problems that are representative of a large class of problems solved using iterative methods are used. We show that a static analysis to determine data dependences in the triangular solve can greatly improve its parallel efficiency. We also show that ignoring symmetry and storing the whole matrix can reduce solution time substantially.
\end{abstract}

\footnotetext{
submitted to: International Journal of High Speed Computing.

'Ph.D. Student at Rensselaer Polytechnic Institute. Troy, NY 12180 and Visiting Research Associate at Research Institute for Advanced Computer Science, NASA Ames Research Center. Woffett Field, CA 94035.

${ }^{1}$ Research Institute for Advanced Computer Science, NASA Imes Research Center. Moffett Field. CA 94035.

${ }^{\circ}$ Work reported herein was supported by Cooperative Agreement $\mathrm{YC}^{\circ} \mathrm{C} 2-38$ - between the $\mathrm{Va}$ tional teronautics and Space Administration (N.t.S. ) and the Cniversities Space Research Association ( CSRA).
} 


\section{Introduction}

We explore different schemes for exploiting the parallelism available in the ICCG method for solving large sparse systems of linear equations on a shared memory computer. All of this work has been conducted on a 12 processor Sequent Balance 21000. We have looked at the efficient implementation of methods for solving triangular systems and at sparse matrix vector multiplication.

An important difficulty in solving general sparse triangular systems is that the available parallelism depends on the zero structure of the matrix, and is therefore not known at compile time. The concurrency is data dependent and can be determined only at run time. We show that by performing a small amount of analysis to determine the data dependences one can drastically improve the parallel efficiency. We permute (reorler) the index set of the recurrence equation for the triangular solve and put the indices in a queue. The processors repeatedly take indices from the queue, perform the associated ralculations, and then take another index until all unknowns have been computed. Data dependences are resolved by semaphores. I semaphorf is a variable that can be operated upon only by synchronizing primitives. We check indices in a shared array that indicate whether each of the unknowns has been computed. If a calculation depends on a piece of data and an entry in the shared array indicates that it has not been computed then the processor performing the calculation must busy wait. Busy waiting is when a processor loops waiting for a flag to change value.

Also, we show that there is a tradeoff between storing the lower triangular part of a symmetric matrix and storing the entire matrix. Storing the lower part to save storage complicates the multiplication since both outer products (which require synchronization) and inner products must be performel. The synchronization overhead slows down this operation.

For our experiments we work with systems of equations in the form they are presented. We do not consider the problem of reordering the rows and columns to enhance parallelism.

The rest of this paper is organized as follows. Section 2 review's related research. Section 3 contains a brief discussion of the ICCG method. Section + discusses how the dependence graph is used to exploit the parallelism in solving sparse triangular systems. Section 5 contains numerical experiments that show it is more efficient to store the whole symmetric matrix than only the upper or lower triangular part. In Section 6 we compare solving a lower triangular system by inner products versus solving by onter products. Section $T$ presents the efficiency of the ICCG method using the techniques described in the previous sections. Section $\&$ dicusses other scheduling methods not used in this paper. Section 9 contains remarks and conclu- 
sions. Ippendix 1 describes the $T$ test ases used in the experiments. Appendis $B$ discusses the architecture of the Sexpmut Balanen 21000 and provides limes for ariblumetic operations and syuchronization primities. In Ippendix ('we show how the time to acress array elements increases as a function of the artay size on the Sequent Batance 21000 .

\section{Related Work}

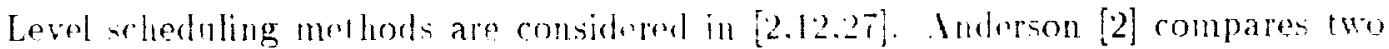
different scheduling methods for solving iparse triangular systems on the Alliant

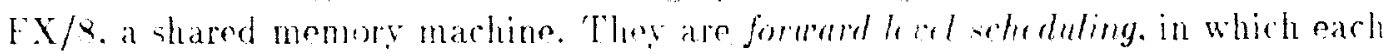
unknown in the triangular solve is computed as arly as posibible. and baskurard to of scheduling. in which rarh unknown is computed at he latest ponsible time. I lovel scheduling approach partitions the loop of the mentrence ofluation into a sequenew of fully parallelized do lonps (levels) separated by global ivelimonizations. He show that the overheal in wheduling tasks to be performerl as late an possible is not worth the time savings.

Baxter $f$. al. [3] compare level wheduling with a solf secheduling method using a hared memory computer. an Fneore Wultimax/320. The self wheduling mothod is a two step procedure to parallalize the recurrence efualion of the triangular solve. First. one performs a ropologiral sort of the dependence graph to permute the index loop. Vext, statically asign elements of the index set to the processors of thr system. Cilobal synchrouizations are avolded by requiring processors to write into specified locations of shared arrays when work on a particular index is completed. Before a variable can he used, a procesion makes sum thal the appropriate values have ben ralculated by bung wrating on a designated walue in the whared memory. They show that self wheduling performs belter than be we wheduling for all but one of their test cases.

The work of Saltz, 1. wl. [2.j is similar wo the work of Bixter. They also compare

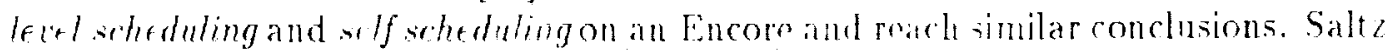
also proposes a unu programming conntruct. downsider which allows compilers to parallelize many problems in which substantial loop. fesel parallelism is arailable but cannot be detecteel bs - tandard compile-timeranalyis.

The lifference between the work presented lere and presions work on triangular

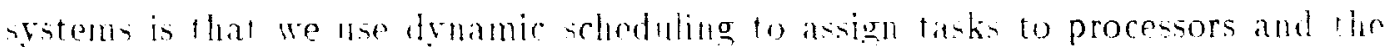
whers 11 se static scheduling.

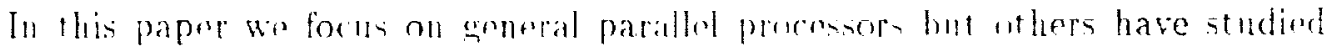
implementations on parallel rector mathinen [15.14.20.26]. Nhditionally saad [21: 
presents a survey of recent research in krylow subsate methods with an emphasin on parallel and vector implomentations.

\section{ICCG Background}

Here we give a brief introduction to the Incomplets Cholesk Conjugate Gradiont [ICCC) method. For lentaled information of its derivation and properties sec references [6.11.13.17,18.2:3]. Th+ Conjugate Gradient (c' $\left.C_{i}\right)$ mellond was proposed by Hestenes and stiefel [13] for the solution of

$$
\mu=b
$$

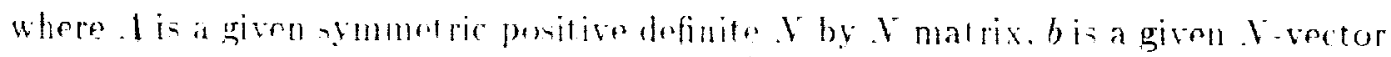
and $x$ is an 1 - vector to be romputed.

Stating from an intial gures $x^{(0)}$, the (') method generates a series of approximate olutions $x^{(k)}$. The converence rate is very poor for ill-conditioned problems [11]. One way to improsw the convorgence is to pre-condition (1) premultiply it by a conditioning matrix and thereby condense the eigenvalue spectrum [1].

1 popular preconditioner is the Incomplete cholesky precouditioner proposent by Vejerink and lau der Vorit [ly]: they perform an approximate Choleskyfactorization $L L^{\Gamma}$ of 1 with zero fill. Equation (1) now becomen:

$$
\left[\left(L^{-1}\right) L^{-1} \cdot\right] x=\left(L^{-\Gamma}\right) L^{-1} b
$$

$L^{-1}$ is not explicitly computed, insterd riangular systems are solved. Each iteration of the IC CG method requires the solution of two sparse triangular systems. a sparse matrix rector product, 3 saxpy's and 2 inner products.

We warn the reader that we use an inconsistont notation here from the rest of the paper. Hore we subscript a vector to indicate that it is a momber of a sequence rather than referring to an individubl alement. The greek letters represent scalars. The [CCG method is bolow:

$$
\begin{aligned}
& x_{0}:=0 \\
& r_{0}:=b \\
& \delta:=z\left\|r_{0}\right\| \text {. } \\
& \text { reperat For } k=1.2, \ldots \\
& \text { Solve } \text { I. }^{\Gamma} \Gamma_{z-1}=r_{k-1} \ln i_{k-1} \\
& f_{k}:=-\frac{T}{4}-1 ; k-1 / 2 T-2 ; k-2 \quad\left(s_{1} \equiv 0\right) \\
& p_{k}:=z_{k-1}+y_{k} p_{k-1} \quad\left(p_{1} \equiv z_{1}\right)
\end{aligned}
$$




$$
\begin{aligned}
& \alpha_{k}:=z_{k-1}^{T} r_{k-1} / p_{k}^{T} \cdot A p_{k} \\
& x_{k}:=x_{k-1}+\alpha_{k} p_{k} \\
& r_{k}:=r_{k-1}-\alpha_{k} A p_{k} \\
& \text { until }\left\|r_{k}\right\|_{\infty} \leq \delta \\
x:= & x_{k}
\end{aligned}
$$

For our codes we choose $\Xi=10^{-b}$ so our iteration stops when the infinity norm of the residual is reduced by 6 orders of magnitude.

\section{Triangular Systems}

At each ICCG iteration we solve the triangular systems

$$
L q=r
$$

and

$$
L^{T} z=\eta
$$

Together, these two operations consume between $30 \%$ and $41 \%$ of the total cpu time required to solve the system on a single processor for our test cases. The percentage depends on the sparsity of $L$ - the more nonzero elements in $L$ the higher the percentage. The remaining time is consumed by sparse matrix-vector products, inner products and saxpy's. These are relatively easy to compure in parallel. Efficient, parallel computation of the triangular solves is necessary to accelerate the entire computation.

The system (3) is solved by

$$
q_{i}=\frac{r_{i}-\sum_{j=1}^{i-1} L_{i, j} q_{j}}{L_{i, i}} \quad i=1, \ldots, .
$$

In the dense case, each $q_{2}$ depends on all $q_{j}, j=1, \ldots i-1$. When $L$ is sparse. each $q_{i}$ depends on a few other $q_{j}$. Another way to look at it is that once some $q_{j}$ has been computed, several other $q$ 's may be computed in parallel. It is possible to perform some simple analysis of the data dependences to determine which elements of $q$ can be computed in parallel and determine which $q_{j}$ s each $q_{i}$ depends on. This information can be utilized to schedule tasks. For example, if $q_{i}$ depends on $q_{j}$ then $q_{j}$ should be scheduled before $q_{i}$. Also, if $q_{j}$ and $q_{k}$ are independent tasks then we may schedule them to be computed in parallel. 


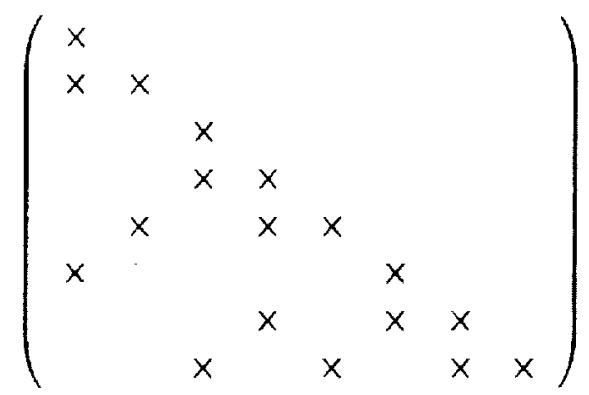

Figilre L: Sparsity Structure of $L$

\subsection{Computing the Dependence Graph}

The problem one faces when exploiting this type of parallelism is that it is data dependent and can only be recognized at run time, not at compile time. It depends entirely on the sparsity structure of $L . L$ is usually read in as input or computed at an earlier stage of the program. The focus in this section and the next one is on lower triangular systems. A similar analysis can be done on upper triangular nlatrices.

Consider solving (3) where $L$ has the sparsity structure shown in Figure 1. Analysis of the structure of $L$ enables us to construct a corresponding directed graph (digraph), the dependence graph $G(L)=(V . E)$. There are $\mathrm{V}$ vertices, $V=\{1, \ldots, V\}$, corresponding to the $V$ rows of $L$ (and $V$ elements of $q$ ). A nonzero eleinent at $l_{i, j}$ means that $q_{i}$ depends on $q_{j}$; i.e.. $q_{j}$ must be calculated before $q_{i}$. Therefore. we define the edges of $G(L)$ as follows: $E=\left\{(j, i) \mid l_{i, j} \neq 0\right\}$. We ignore the loops corresponding to the diagonal elements of $L(C r(L)$ is acyclic). The depth of a vertex $v_{i}$ is 0 if it has no predecessors otherwise the depth of $v_{i}$ is the length of the longest directed path in $G(L)$ whose origin is a vertex of depth 0 and terminus is $v_{*}$.

The dependence graph of $L$ is shown in Figure 2. All nodes at depth 0 can be computed immediately. They have no dependences. $q_{1}$ and $q_{3}$ can be solved directly. Once $q_{3}$ is computed we can solve for $q_{t}$. After $q_{1}$ is computed we can solve for $q_{2}$ and $q_{6}$ in parallel. The unknowns $q_{2}$ and $q_{6}$ depend only on $q_{1}$. Once $q_{2} \cdot q_{6}$ and $\eta_{4}$ have been computed, we can solve for $q_{5}, q_{i}$ in parallel. Vertices that have equal depth represent independent tasks. The fact that $q_{2}$ and $q_{6}$ can be computed as soon as $q_{1}$ has been compited. even if $q_{3}$ has not been completed, illustrates the difference between lert sherluling methods and self sherluling methods. If tel 


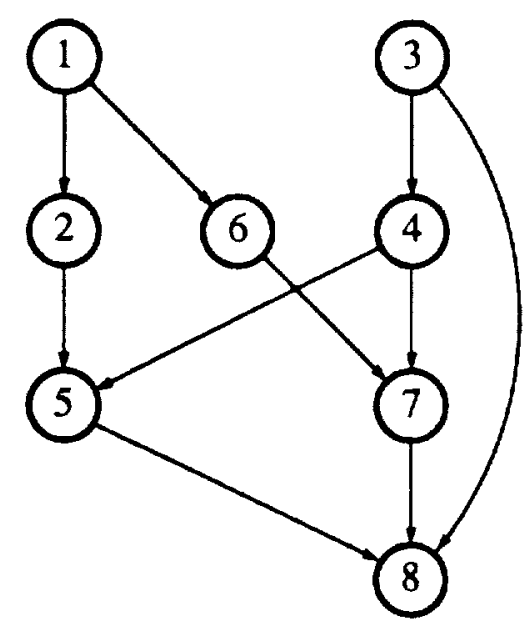

Figure 2: Dependence Graph of $L$

scheduling computes tasks corresponding to vertices of equal depth in $G$ in parallel. All tasks at a certain depth must be completed before tasks at the next level can be started. Global synchronizations are used to separate tasks at different depths, self scheduling allows tasks to start as soon as their associated dependences have been computed.

\subsection{Permuting the Index Set}

The index set for the sequential solution of equation $(5)$ is $i=1, \ldots v$. To exploit the parallelism in the forward solve we reorder the index set according to the depth of each index in the dependence graph. A vertex of a certain depth is put in the permuted set before all vertices of greater depth. We define postion $(k)$ to be the number of elements in the premuted index set that precede $k$. If two vertices $r$ and $v_{3}$ have equal depth then we put $v_{i}$ in the permuted index set before $v_{3}$ if

$$
\max _{n}(\operatorname{position}(n))<\max _{m}(\operatorname{position}(m)) \text { such that }(n, i),(m, j) \in E \text {. }
$$

If

$$
\max _{n}(\operatorname{position}(n))=\max _{m}(\operatorname{position}(m)) \text { such that }(n, i),(m, j) \in E
$$

then $n=m$ and $v_{i}$ is placed in the list before $y_{j}$ if $i<j$. This is a side effect of the sequential traversal of the data structure for $L$. 
We all this permuted index set fwd.schedule. For example, the permuted index set from the dependence graph in Figure 2 is

$$
\text { fwd_schedule }=\{1,3,2,6,4,5,7,8\}
$$

Note that 6 appears before 4.6 is a descendent of 1 and 4 is a descendent of 3 and position $(1)<\operatorname{position}(3)$.

One way to compute the fwd_schedule list is outlined here. First, as the matrix is assembled or read in, construct an array of length $l$. called the ready array such that ready[i] is the number of nonzero elements in row $i$ of $L$. We then scan the entries of the ready array looking for entries with a value of 1 . If ready(i) $=1$ then $q_{i}$ can le solved for directly. This entry is put in a queue, $Q$. When we have inserted all entries with value $l$ in $Q$ we start the following loop. We follow the notation used in [1] for operations on a quete. A que u€ is a sperial kind of a list. where items are inserted at one end (the rear) and deleted from the other end (the front).

fwd_schedule $=$ nil

While $($ empty $(Q) \neq$ true $)$

L. $i:=$ fronl $(Q)$

2. dequfue(Q)

3. append $\mathrm{i}$ to fwd_schedule list

4. for each nonzero element $I_{k_{i}}$

(a) $\operatorname{ready}(k):=\operatorname{ready}(k)-1$

(b) if $(\operatorname{read} y(k)=1)$ then enqueue $(Q, k)$

The dependence graph is not explicitly computed but the information it represents is implicit in rearly and the ordering of fwd_schedule. We require two integer arrays of length $V$ to hold $f$ wd schedule and ready. This additional storage is small relative to the storage for $A$. $L$ and the other $V$-vectors needed for ICCG.

Equation (4) is also solved with a permuted index set. which we store in the array back_schedule []. It is computed by analyzing the dependence graph of $L^{T}, C_{r}\left(L^{T}\right)$. in a manner similar to that used to compute fud_schedule. Let $G\left(L^{T}\right)=\left(V_{T} \cdot E_{T}\right)$, $V_{T}=I$ and $E_{T}=\{(j, i) \mid(i, j) \in E\} . C\left(L^{T}\right)$ is the same as $G(L)$ with the direction of the edges reversad. For the example shown in Figure 1. the schedules for solving the upper and lower triangular systems are the reverse of each other. This is not true in general. Suppose that we have the same lower triangular matrix as in Figure 1 except $L_{8.1}$ and $L_{8,8}$ are the only nonzero elements in row 2 of $L$. Then. $v_{8}$ will 


\begin{tabular}{|r|c|c|c|c|c||}
\hline case & fwd_schedule & fwd solve & back_schedule & bck solve & $. \mathrm{Ix}=\mathrm{b}$ \\
\hline 1 & .12 & .18 & .11 & .16 & 1.89 \\
2 & .06 & .06 & .05 & .06 & 2.45 \\
3 & .05 & .05 & .04 & .04 & 3.07 \\
4 & .07 & .08 & .06 & .08 & 3.21 \\
5 & .10 & .11 & .09 & .10 & 8.85 \\
6 & .25 & .30 & .23 & .28 & 8.52 \\
7 & 3.12 & 3.25 & 2.09 & 2.48 & 6.5 .5 .39 \\
\hline
\end{tabular}

Table 1: Time in seconds to compıte task schedules vs. single sequential triangular solve and solving $\mathrm{Ax}=\mathrm{b}$ in parallel

be depth 1 in $C(L)$ and 8 will be the $6^{\text {th }}$ element in fwd_schedule[]. But, rs will be at depth 0 in $G\left(L^{T}\right)$ and the first elenent in back_schedule [].

The time to compute the permuted index sets is a little less than the time to compute a single sequential triangular solve and a small fraction of the time to solve (2) in parallel. The time in seconds to compute the forward and backward schedules for the test cases is shown in Table 1. We compare the time to compute the fwd_schedule and the back_schedule lists with the time to sequentially compute one forward and backward solve and with the time to solve $A x=b$ in parallel.

\subsection{Forward Solve}

We solve (3) as follows. $L$ is stored by columns and the forward solve is computed as a set of outer products. fwd_schedule is the list of indices which correspond to elements of $q$ to be computed. It is treated as a queue of tasks to be executed by the pool of processors. Let there by $P$ processors. Initially, the first $P$ indices in the queue are assigned one to each processor. Let $i$ be the index a processor gets from the queue. Before we compute each forward solve we set fwdready [] to be the number of nonzero elements in each row of $L$. If $f$ wdready[i] $\neq 1$, then the processor must busy wait. else, compute $r_{i}=b_{i} / L_{i, i}$. Sext, compute $r_{j}-=L_{j . i} q_{i}$ and decrement fwd_ready $[j]$ for all nonzero elements $j$ of column $i$ of $L$. Finally, if the queue is not empty get the next task.

For the triangular solve, we experimentally compared three different techniques for parallelizing the code. We call the first method dynamic scheriling (DS). The elements of $q$ are assigned to processors in order, from 1 to $Y$. They are computed as soon as the data they depend on is ready to be used. The data illustrate that 
poor performance may be expected if the index set is left in its original order.

The second technique is due to Baxter $\epsilon t$. al. [3]. We call this technique reordered static scheduling (RSS). They use the reordering strategy above but employ a static assignment of tasks from the permuted index set to processors. Let $P$ be the number of processors. Processor $i, 1 \leq i \leq P$. gets tasks $i+P \times j$ for $j=0, \ldots,\left\lfloor\frac{v-1}{p}\right\rfloor$. It has the advantage that for every iteration each processor will solve for the same values of $q$. This characteristic is especially noticeable for small problems when the entire problem fits in the local memory (or cache) of the processors. But this may not be very good at load balancing. If there are wide variations in the number of nonzero elements in the rows/columns of the matrix then the static mapping may cause unnecessary husy waiting. This variation arises in many different situations: non-uniform discretizations, adaptively refined meshes, or mixed elemeut types (triangular and quadrilateral elements in the same grid) for instance.

The problem with roorderfd static srheduling is that the position of the task in the schedule is determined solely by its dupth in the dependence graph. The strategy does not consider the amount of time nceded to perform the task. It is possible that a static assignment of tasks to processors could result in uneven distribution of work and lower or less throughput.

The third technique is called rfordered dynamir scheduling (RDS). We reorder the index set as above. but we put the indices (tasks) in a queue rather than statically mapping them to processors. The first processor done with the work initially assigned to it takes the next job from the front of the queue. This is done to reduce the time spent busy waiting due to potential load imbalance. There is an additional expense of maintaining a global pointer (m_rext() on the Sequent) to the first element in the quene.

The $\mathrm{C}$ cole for reodered dynamic scheduling is shown in Figure 3. m_next () is the system function which increments a global counter and returns its current value. fwd_schedule[] is our permuted index set for the forward solve. As suggested by Duff, et. al. [i], we store the columns as packed sparse vectors held contiguously in the array 1[] . The row numbers of the corresponding nonzero entries held in 1[] are hell in the integer array rownum[]. The integer array start [i] points to the start of column $i$ in array 1 [] containing the nonzero elements of matrix $L$. In fact 1 [start $[i]]$ is the diagonal element $L_{i, i}$. The global variables unknowns and tot_nonzero hold the number of rows in $L$ and the total nonzero elements in $L$ respectively start [unknowns +1$] \equiv$ tot_nonzero +1 .

Once we have gotten a task from the queue, we check whether all of the data it needs are rnady. This is done by looking at the value of the fwd_ready [i] array containing the number of direct dependences for row $i$. If the value is greater than 


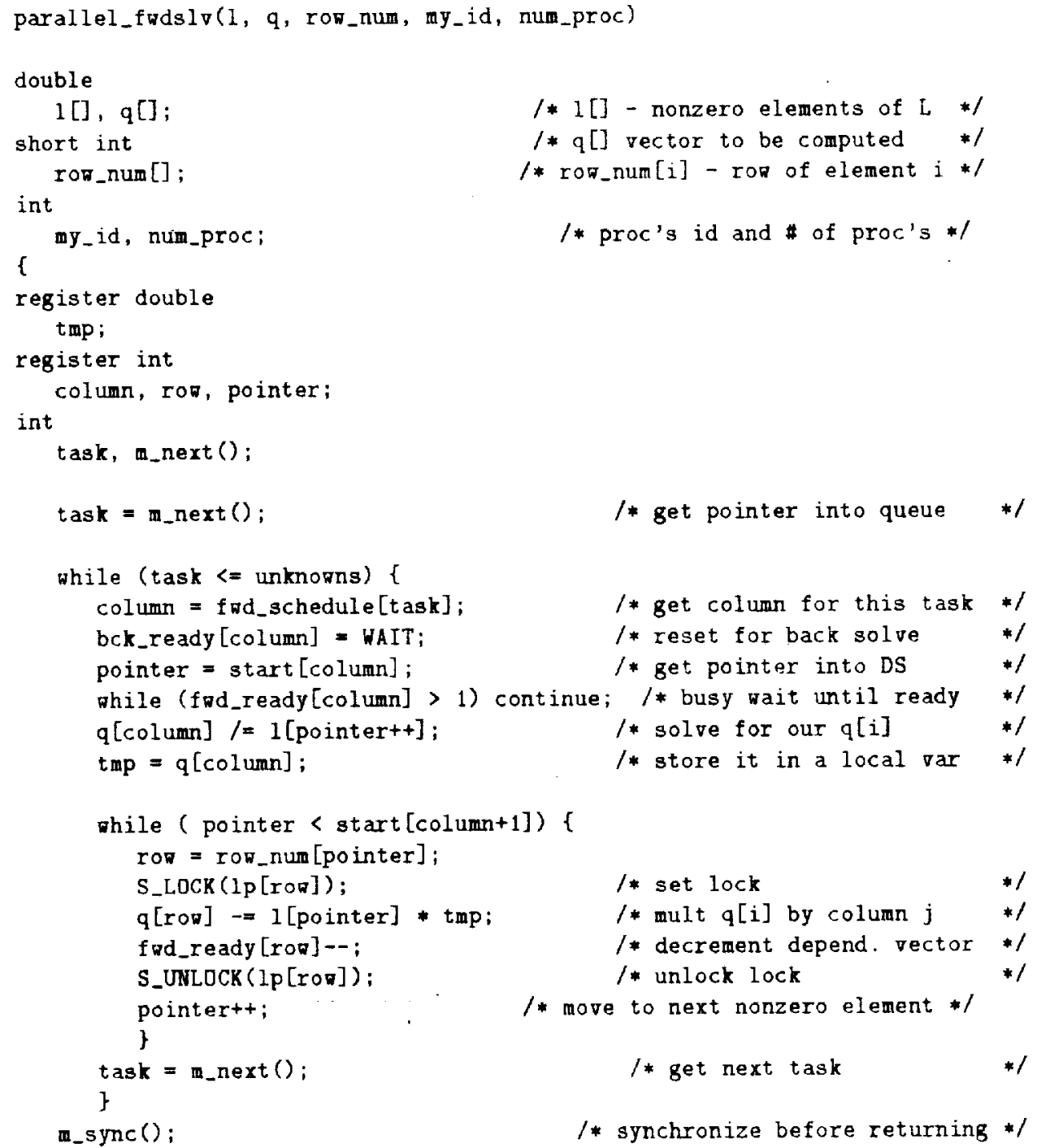

Figure 3: Procedure for Parallel Forward Solve 


\begin{tabular}{||c|c|cc|cc|cc||}
\hline \multirow{3}{*}{ Case } & \multicolumn{7}{|c||}{ Type } \\
\cline { 2 - 8 } & Sequential & \multicolumn{2}{|c|}{ DS } & \multicolumn{2}{c||}{ RSS } & \multicolumn{2}{c||}{ RDS } \\
\cline { 2 - 8 } & $t_{3}$ & $t_{p}$ & effir. & $t_{p}$ & effic. & $t_{p}$ & effic. \\
\hline 1 & 2.97 & 1.61 & .17 & .73 & .34 & .79 & .32 \\
2 & 2.30 & .78 & .30 & .35 & .42 & .77 & .30 \\
3 & 3.29 & 1.07 & .26 & .72 & .38 & .99 & .28 \\
$t$ & 3.24 & 1.54 & .21 & .90 & .36 & 1.03 & .31 \\
.5 & 11.32 & 3.48 & .27 & 2.13 & .44 & 2.92 & .32 \\
6 & 13.29 & 11.04 & .10 & 2.73 & .41 & .3 .29 & .34 \\
7 & 66.21 & 11.21 & .13 & 219.46 & .25 & 206.81 & .27 \\
\hline
\end{tabular}

Table 2: Time in seconds and efficiency of parallel forward solve on 12 processors relative to sequential rode

I then we busy writ. When fwdready [i] is equal to 1 the dependences for $q_{i}$ have beon satisfied and we can compule $q_{i}$. We set $q_{i}=q_{i} / L_{i, i}$ and then loop over the nonzero elements in column $i$ below the diagonal, computing $q_{j}=q_{j}-L_{j . i} \times q_{i}$. Then we der rement the value of the fwd_ready $[j]$ array to indicate that one dependence for $q$ has been atinfied. The array $q$ and the fwdready array are shared and access to individual olements must be synchronized using the system calls S_LOCK() and S.UNLOCK(). These synch ronization procedures are called once for each nonzero off-diagonal element in the lower triangular matrix each iteration. This locking and unlocking operation takes about half of the time in the forward solve routine when the matrix is stored by columns. We also reset bck ready [] for the next back solve operation.

In Table 2 we show the results for the three methods explained above on seven test problems. This is the time spent during the iterative solution of $1 x=b$ doing forward solves. All times are measured in seconds. We also include the sequential time for for each problem. $t_{s}$. The sequential time given is the best sequential code we could write running on one processor; there are no parallel constructs or syuchronizations used. Parallel code running on 12 processors of the Sequent took time $t_{2}$. We measure efficiency as

$$
\text { effic. }=\frac{t_{s}}{t_{p} \times \# \text { proc }}, \quad \# \text { proc }=12
$$

The DS timings are included for comparison to illustrate the benefit of computing the dependence graph and permuting the index set. We see that both RSS and 


\begin{tabular}{||r|c|c||c||}
\hline Case & $t_{R D S}-t_{R S S}$ & Predicted & \# iterations \\
\hline 1 & 0.05 & 0.06 & 16 \\
2 & 0.22 & 0.11 & 41 \\
3 & 0.29 & 0.14 & 68 \\
4 & 0.13 & 0.13 & 45 \\
5 & 0.79 & 0.47 & 101 \\
6 & 0.44 & 0.44 & 43 \\
\hline
\end{tabular}

Table 3: Time Difference between RDS and RSS vs. Estimated time in seconds

RDS are significantly better than DS, sometimes more than twice as efficient. The RSS method performs better than RDS in all but the last problem. In the first Test Case, RSS and RDS the two took almost the same time, and half as long as dynamic scheduling. In the last case, RDS was more efficient than RSS despite the calls to the global counter. This has several possible explanations. First, the number of nonzero elements in each column was more in the first and last cases than in the second through sixth cases. Thus. the relative overhead associated with the global counter versus the amount of work to do per call is less. In the last case, the number of nonzero elements per column raried between 8 and 40 . Cood load balancing is especially important in this case for increased throughput. Statically assigning tasks to processors by their depth in the dependence graph alone (as in RSS) cannot achieve this. There must be some way to account for the amount of work to be done in each task, not just the dependences of the task. The RDS method performs better at this than the RSS method as shown in Test Case 7 .

When the problems have a regular sparsity structure (most of the columns/rows have the same number of nonzero elements) the time to compute each $q_{i}$ is roughly the same and the load is balanced as long as each processor gets roughly the same number of $q_{1}$ 's to solve for. Test Cases $1-6$ have a regular sparsity structure and thus the RSS method performed slightly better. The main contribution to this difference is the fact that in the RDS technique a glohal counter is used to maintain the quene of tasks. It takes about $50 \mu$-seconds for each call and this is done before each $q_{i}$ is computed. A prediction for the time difference when there is a regular sparsity structure is

$$
t_{R D S}-t_{R S S} \approx(50 \mu-\operatorname{seconds})(\# \text { iterations })\left(\frac{\# \text { inknowns }}{\# \text { proc's }^{\prime}}\right) .
$$

In Table 3 we compare the actual difference with the prediction for the first 6 cases. The right most column shows the number of iterations for convergence for each test 
case. This model gives an estimate of the size of the difference that is correct to within a factor of two.

\subsection{Backward Solve}

The backward solve is sinilar to the forward solve, but there are subtle differences in implementation. To solve (4) we carry out the computation as a series of inner products rather than outer products. $L^{T}$ is accessed by rows since we store $L$ by coliunns.

An outline of our back solve procedure follows. Just as in the forward solve. we have a list of permuted indices back_schedule[]. It is computed in a manner

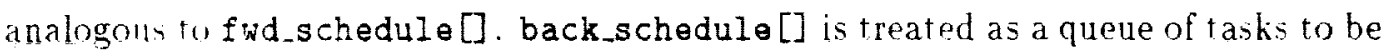
computed by the processors. bck_ready [] is initialized to the value "WAIT"'. For som $j$. if bck ready $[j]=$ WAIT then this indicates that $z[j]$ has not been computed Fet. Fach processor gets an indes from the queue as it begins the back solve. Let $i$ be the index that ione processor gets. For each nonzero element $j$ in row $i$ of $L^{T}$. check bck_ready $[j]$. If bck ready $[j]=$ WAIT, then busy unit. Else. compute $z[i]-=L_{i, j}^{T} z[j]$. When all nonzero off-diagnoal elements in row $i$ have been used we calculare $z[i]=z[i] / L_{i, i}^{T}$ and set bck_ready $[i]=$ "DONE'". The value DONE indicates that the element of $z[]$ is computed. Finally, if the queue is not empry get the next index.

The $\mathrm{C}$ code for this techuirue is shown in Figure 4. As in the forward solve routine we compute the new vector in place, overwriting the previous entries of $z[] .1[]$ is the array containing the nonzero elements of the rows of the upper triangular matrix. The beginning of row $i$ is pointed to by the array start [i]. To move across the nonzero elements of row $i$, from right to left, we start at pointer $=$ start $[i+1]-1$. start $[i+1]$ points $10 L_{i+1, i+1}$ in 1[] and start $[i+1]-1$ points to the right-most nonzero element in row $i$. The bck_ready[] array is set to "BUSY" during the provious forward solve. Therefore. if bck_ready [j] = BUSY. then $z$ [j] has not been computed yet in the back solve. To reset fwd_ready [j] for the next forward solve we set $f w d_{-} r e a d y[j]=f$ wddepend $[j]$. fwd_depend $[j]$ is the num. ber of nonzero elements in row $j$ of $L$. To indicate that $z[j]$ has been computed in the back solve we set bck ready [j] to "DONE". The back_schedule[] array contains the index set that has been pernuted appropriately for the back solve operation. Finally, we set a barrier m_synch() to synchronize all processors at the end of the procedure before returning.

There is no need to do the locking and unlocking as in the forward solve routine. This procedure only writes to three hared arrays fwdready [], bck_ready []. and $z$ []. Fach location in read by many procesiors but only writen to by one procesor. 


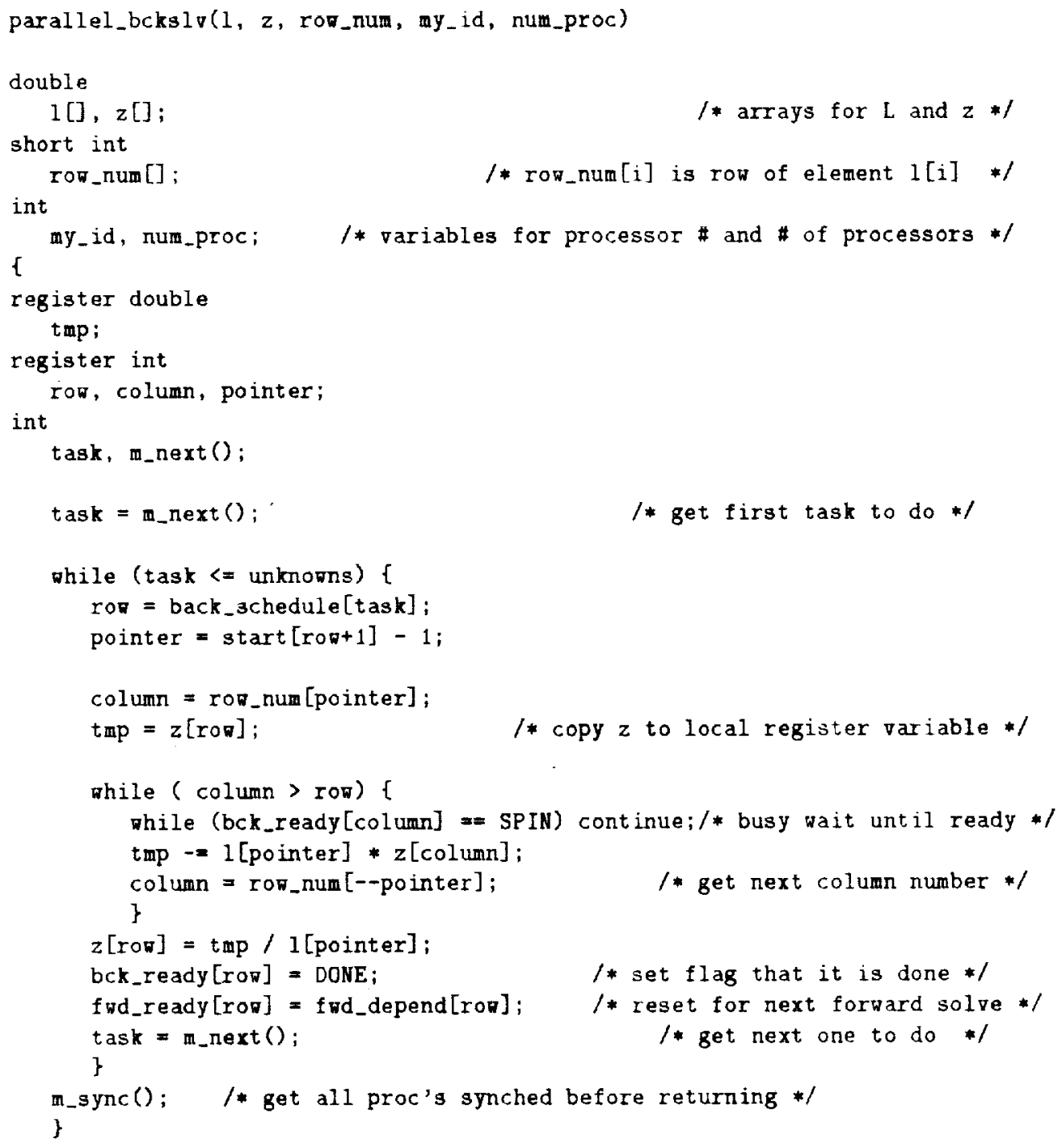

Figure t: Procedure for Parallel Backward Golve 


\begin{tabular}{||c|c|cc|cc|cc||}
\hline \multirow{3}{*}{ Case } & \multicolumn{7}{|c||}{ Method } \\
\cline { 2 - 8 } & Sequential & \multicolumn{2}{|c|}{ DS } & \multicolumn{2}{c||}{ RSS } & \multicolumn{2}{c||}{ RDS } \\
\cline { 2 - 8 } & $t_{s}$ & $t_{p}$ & effic. & $t_{p}$ & effic. & $t_{p}$ & effic. \\
\hline 1 & 2.70 & .95 & .21 & .38 & .58 & .15 & .50 \\
2 & 2.65 & .67 & .33 & .42 & .53 & .62 & .36 \\
3 & 3.07 & .83 & .31 & .49 & .52 & .78 & .33 \\
4 & 3.56 & 1.01 & .29 & .5 .5 & .54 & .81 & .37 \\
5 & 10.75 & 2.63 & .34 & 1.54 & .58 & 2.56 & .35 \\
6 & 12.38 & 7.18 & .14 & 1.72 & .60 & 2.35 & .44 \\
$i$ & .507 .90 & 103.92 & .41 & 85.44 & .50 & 85.80 & .49 \\
\hline
\end{tabular}

Table t: Time in seconds and efficiency of parallel backward solve on 12 processors relative to sequential code

Co locking is reyuired in this situation. The inner product form of the triangular solve therefore has much less overhead.

In Table $t$ we rompare the three methods for the backward solve. DS is clearly slower than the other two. It is only included for comparison. We see that the RSS method performs better than the other methods. Just as for the forward solve. the time difference is due to the fact that in RDS a global counter is required to maintain the quele of tasks. The difference is very pronounced for problems 1-6: since there is very little work to do to compute each $z_{i}$; i.e., there are only a few nonzero off-liagonal elements in each row/column. The efficiency of RSS and RDS are almost identical for Test Case 7 . The load balancing that is provided in RDS makes up for the overhead of using the global counter. The amount of work to be done to compute some $z_{i}$ is directly related to the number of nonzeros in row $i$ of $L$. The amount of work per task affects the load balancing. Test Case $T$ has the most variation in the number of nonzero elements in its rows (and columns). As the variation increases so does the need to account for this in the scheduling of tasks.

\section{Matrix-Vector Product}

In this section we discuss the implementation of sparse matrix-vector products on the Sequent. We show that it is more efficient to store the whole symmetric matrix by rows rather than trying to save storage and storing only the lower or upper triangular half. This is true for both the sparse matrix-vector product and the 
triangular solves. To compute a general symmetric sparse matrix-vector product $A x=b$ on the Sequent it is more efficient to store all of $A$ by rows than to store only the lower triangular part by rows (or columns).

When a sparse symmetric matrix is stored as a lower triangular matrix by columns or rows (or if the upper triangular matrix is stored by columns or rows) the multiplication must be carried by a combination of inner and outer products. The implementation becomes complicated and requires synchronization to protect elements of shared arrays from being modified by more than one processor at a time.

An implementation of a symmetric sparse matrix-vector product written in $\mathrm{C}$ is shown in Figure 5. For this example, only the nonzero elements of the lower triangular part of $A$ are stored (by columns). Firit. each processor initializes a portion of the array $b[]$ to be zero. Next, each processor gets a column of the data structure. This is a column of the lower triangular part of the matrix and a row of the upper triangular part. A column in the lower part, say column $i$, is multiplied by element $x[i]$. The product is accumulated into the shared array $b[]: b[j]+=a[$ pointer $] \times x[i]$. To be sure that only one processor is writing to $b[j]$ at a time we must use the system synchronization routines SLOCK() and S_UNLOCK (). Vext we multiply the element of colurnn $i$ by $x[j]$ and add the product to the local rariable inner_prod. When we have exhausted all elements of the upper triangular row, we add the local inner product into the shared array b [] using the appropriate locks. In essence, we accumulate inner products locally and add outer products globally. This approach requires two system synchronization calls per nonzero element in the lower triangular part of $A$. Even though the probability of collision is small since we are dealing with a sparse matrix, this has to be done to insure that only one processor updates an element of $b[]$.

A procedire for computing a general sparse matrix-vector product where the full $A$ is stored by rows is much simpler and is shown in Figure 6. Each processor computes a set of inner products. The processors dynamically get an element of $b[]$ to compute using the system global counter m_next() The array row_start [] is an array holding the starting point for each row as it is stored in the data structure. The inner product of each row with $x$ is computed and stored in $b[]$. This algorithm requires no synchronization since the work is divided into non-overlapping groups of rows.

In Table $;$ we compare two methods for parallel computation of the sparse matrix vector product with the time it takes to compute it sequentially. The first method, "Symmetric", is the symmetric code from Figure i. It takes advantage of symmetry and stores only the lower triangular part of the matrix. The second method, "Vosynch", is the same algorithm but we have commented out all of the synchronization calls to S_LOCK() and S_UNLOCK(). The answer we get is incorrect 


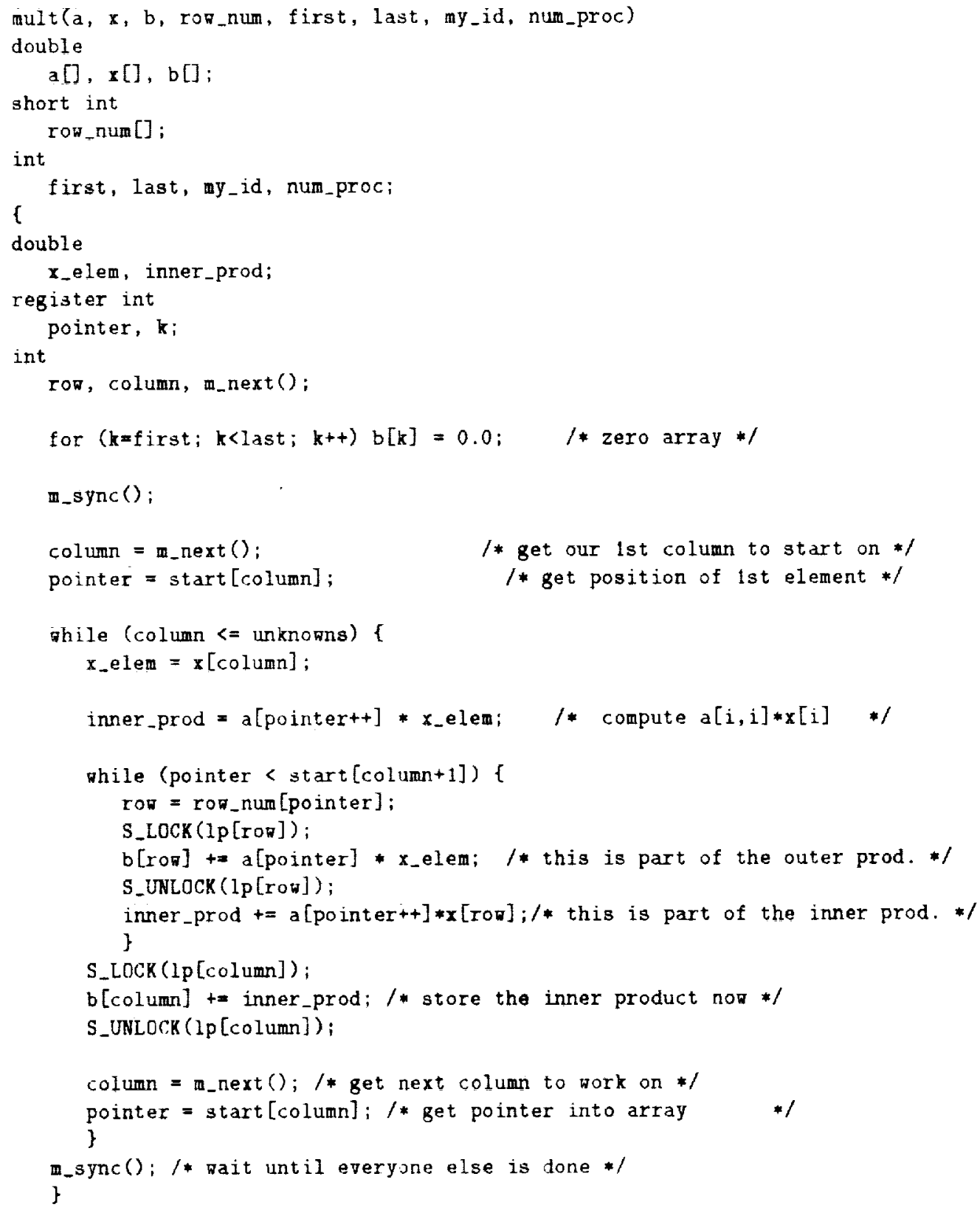

Figure i: Code for Simmunic Spara Matrix-Vector Product 


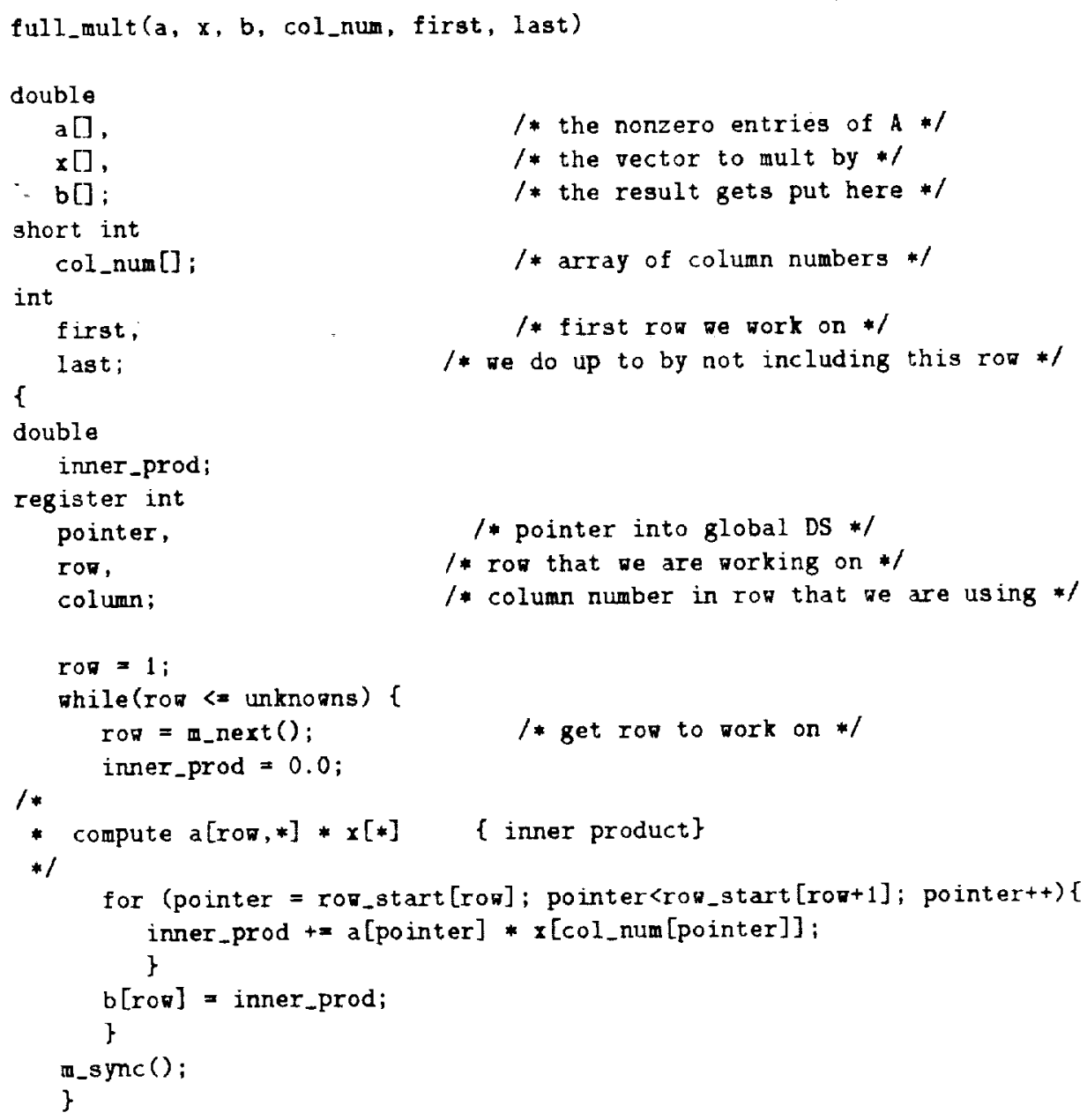

Figure 6: Code for Full sparse matrix-vector product 


\begin{tabular}{|c|c|c|c|c|c|c|c|}
\hline \multirow{3}{*}{ Cathe } & \multicolumn{7}{|c|}{ Wethod } \\
\hline & \multirow{2}{*}{$\frac{\text { Sequential }}{t_{s}}$} & \multicolumn{2}{|c|}{ Symmet ric } & \multicolumn{2}{|c|}{ Nosynch } & \multicolumn{2}{|c|}{ Full } \\
\hline & & $t_{p}$ & effic. & $t_{p}$ & effic. & $t_{p}$ & effir. \\
\hline 1 & 5.86 & .91 & .34 & .58 & $.8+4$ & .56 & $x_{i}^{-}$ \\
\hline 2 & 5.29 & .99 & .15 & .59 & .75 & .63 & .70 \\
\hline 3 & 6.03 & 1.06 & $4 i$ & .71 & וז. & .66 & .76 \\
\hline$t$ & 7.23 & 1.35 & .45 & הi & . & .76 & .9 \\
\hline 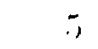 & 21.20 & 3.64 & .49 & 2.41 & .73 & 2.27 & .78 \\
\hline ij & 13.29 & 3.82 & .56 & 2.35 & .91 & 2.55 & .84 \\
\hline$i$ & $1270 . \times 0$ & 321.8 .7 & .33 & 226.13 & .47 & 207.16 & .51 \\
\hline
\end{tabular}

Table j: Time in seconds and efficiency of parallel Sparse Matrix-Vector product on 12 pronsisor, relative to seculuential code

but we stop after the same number of iterations. This is to show the impact of the synchronization. It also gives us a lower bound on the time for this method of matrix-rector product. The last method, "Full", is the times from the code in Figure 6.

We see that storing the full matrix is best. Timing and efficienry is better than $70 \%$ except for large problems. We expect this. since matrix-vector products are very parallel computations. If we look at the difference between the parallel limes of the Symmetric and Nosynch columns it is clear that to use the system synchronization calls adds almost $50 \%$ to the cost of the computation. But, even without the synchronication. we sec that the Full method is better than the Nos:nch method. From this we conclude that there is no advantage in storing only half of a symmetric matrix for parallel computation of the sparse matrix-vector product on this machine.

An alternative to sparse matrix rector multiplication romputed as inner or outer products is proposed my. Melhem [19] where he siggests a general technique of using striped matrix storage.

\section{Triangular Solve Revisited}

The decision to store the full matrix I affects ot her part s of the code. We also stored the full preconditioner as two triangular matrices. $L$ and $L^{T}$. both by rows. The new values for the timings of the triangular wolves are compared with the old values in 


\begin{tabular}{||r|cc|cc||}
\hline \multirow{2}{*}{ Problem } & \multicolumn{4}{|c||}{ Method } \\
\cline { 2 - 5 } & \multicolumn{2}{|c|}{ Symmetric } & \multicolumn{2}{|c|}{ Full } \\
\cline { 2 - 5 } & fwd & bck & fwd & bck \\
\hline 1 & .73 & .38 & .44 & .12 \\
2 & .55 & .42 & .41 & .42 \\
3 & .72 & .49 & .53 & .48 \\
4 & .90 & .55 & .60 & .58 \\
5 & 2.13 & 1.54 & 1.58 & 1.51 \\
6 & 2.73 & 1.72 & 1.79 & 1.70 \\
7 & 206.81 & 85.80 & 200.52 & 216.63 \\
\hline
\end{tabular}

Table 6: Time in seconds for Triangular Solve on 12 processors

Table 6. The columns labeled "Symmetric" are for storing only the lower triangular half of the symmetric matrix. The columns under "Full" are the timings for storing both the upper and lower triangular matrices of the preconditioner by rows. The forward solve is faster because it uses inner products. There is no synchronication for every element of $L$, only one for each row. We cannot, however. explain the data from Case 7 .

\section{$7 \quad$ Parallel Efficiency of ICCG}

In Table 7 we show the time required to solve (1) for each implementation. assuming the preconditioner has be previously computed. In the first six cases it is clear that storing the full matrix is better than storing only its lower triangle. The efficiency is near or above $60 \%$ for the entire code. This is a very reasonable level and what we expected. But, for the seventh case, the code was not efficient. In Appendix C we show how the time to access array elements increases as a function of thr array size and discuss the time for Test Case 7 .

\section{Scheduling}

Other scheduling methods not considered here are discussed in [9.10.14.22]. The general problem is to schedule a set of partially ordered tasks onto a multiprocessor system so that the time required to complete the tasks is miminized. This problem is known to belong to the class of "strong" XP-hard problems. The work by $[9.10 .22]$ 


\begin{tabular}{||c|c|cc|cc|cc||}
\hline \multirow{3}{*}{ Case } & \multicolumn{7}{|c||}{ Method } \\
\cline { 2 - 8 } & Sequential & \multicolumn{2}{|c|}{ Symmetric } & \multicolumn{2}{|c|}{ Nosynch } & \multicolumn{2}{|c|}{ Full } \\
\cline { 2 - 8 } & $t_{s}$ & $t_{p}$ & effic & $t_{p}$ & effic. & $t_{p}$ & effic. \\
\hline 1 & 15.26 & 2.54 & .50 & 2.29 & .55 & 1.89 & .67 \\
2 & 17.80 & 2.97 & .50 & 2.67 & .55 & 2.45 & .60 \\
3 & 21.00 & 3.89 & .45 & 3.48 & .50 & 3.07 & .57 \\
4 & 22.52 & 4.16 & .15 & 3.43 & .54 & 3.21 & .58 \\
5 & 72.39 & 10.83 & .56 & 9.58 & .62 & 8.8 .5 & .68 \\
6 & 78.09 & 11.01 & .59 & 9.64 & .67 & 8.52 & .76 \\
7 & 2811.14 & 6.55 .39 & .36 & 609.36 & .38 & 660.19 & .35 \\
\hline
\end{tabular}

Table 7 : Time in seconds and efficiency of Total IC'C'C' code on 12 processors relative tusartuential rodm

presents bounds on the number of processors required to compute the tasks in a minimum amonnt of time and hounds the time to compute the tasks with a fixed number of processors. Also. in [10]. bounds on the ratio of times for two different feasible schedules are given.

Kasahara and Varita [1t] present two different scheduling methods. CP/MISF and DF/IHS. ( P/MISF stands for critical path/most immediate successors firs and DF/IHS , tands for depth first/implicit heuristic searrh. The primary difference between the two is that the former schedules tasks as soon as possible and the latter schedules tasks as late as possible. Both require sorting of tasks at the same level according to the number of predessors they have and both are $O\left(X^{2}\right)$ algorithms. where.$T$ is the number of vertices in the dependence graph.

We do nut use either of these srheduling techniques. Sorting the tasks at each level is exponsive. We choose a sheduling method that is not optimal but requires very low owrhead.

\section{Summary}

We have discussed different approaches for exploiting parallelism in the ICCG method for solving large sparse s.mmetric positive definite słstems of equations on a shared memory parallel computer. Wi showed that performing a small amount of analysis to determine the data dependences can drastically improve the parallel efficiencr: Additionally, when the sparsity itructure of a triangular matrix was regular then 
a reordered static scheduling method performed more efficimlly than a reordered dynamic scheduling method. Finally, we showed that for the Secuent it was more efficient to store the whole symmetric matrix by rows rather than only the upper or lower triangular part. The code for a full matrix was simpler and required less synchronization overhead.

\section{References}

[1] A. Aho, J. Hopcroft and J. Cllman. Data Structures and Algorithms. AddisonWesley, 1983.

[2] E. Anderson. Parallel Implementation of Preconditioned Conjugate Ciradient Methods for Solving Sparse Systems of Linenr Systems. Technical Report 805, Center for Supercomputing Research and Development. Cniversity of Illinois at I.rbana-Champaign. Crbana, Illinois, August 1982.

[3] D. Baxter. J. Saltz, M. Shultz, and S. Eisenstat. Preronditioned Kryloc Solvers and Wethods for Runtime Loop Parallelization. Technical Report TR-65.5. Department of Computer Science, Yale University, New Haven (T, October 1988.

[4] G. Bedrosian. FORTRA.V Subroutine Package for Soluing Largf. Sparse, Symmetric Linear Systems. Technical Report \$4CRD284, General Electric Co., Corporate Research and Development Center, 1984.

[5] G. Bedrosian. Private communication. 1986.

[6] P. Concus, G. H. Golub, and D. P. O Leary. A generalized conjugate gradient method for the numerical solution of elliptic partial differential equations. In Gene H. Golub, editor, Studies in Vumerical Analysis. pages 179-198. The Nathematical Association of America, 1984.

[i] I. S. Duff, A. M. Erisman, and J. K. Reid. Direct Methods for Sparse Matrices. Clarendon Press, Oxford, 1986.

[8] I. S. Duff. R. G. Grimes, and J. G. Lewis. Sparse Matrix Problems. Technical Report CSS 191. Harwell Laboratory, October 1987.

[9] E. B. Fernandez and B. Bussell. Bornds on the number of processors and time for multiprocessor optimal schedules. IEEE Trans. Comput., c-22(R): $745-751$, Aug 1973. 
[10] M. R. Garey and R. L. Graham. Bounds for multiprocessor scheduling with resonre constraints. SLA.H J. Comput.. $1(2): 185-200$. 1975.

[11] C. H. Golub and C. F. VanLoan. Iatrix Computations. Johns Hopkins Cnirersity Press. Baltimore. Maryland, 1983. Second Priuting.

[12] 1. Grembaum. Solling Triangular Linear Systems Lsing FORTR.1.V with Parallel Extensions on the . YT Cltrucomputer Prototype. Technical Repurt 99. Courant Instinute. New York Cniversity, New York. NY, 198-.

[13] M. R. Hestenes and E. Stiefel. Werhods of conjugate gradients for solving linear systems. J. Res. Thtionul Burrou of Standards, (19):409-436. 1952.

[1.4] H. Kasahara and Varita S. Practical multiprocessor scheduling algorithms for efficient parallel processing. IEEE Trans. Comput.. c-33(11):1023-1029, Yov $19 \times 4$.

[15] 1. Koniges. Purallel Processing of a Preconditioned Biconjugate Gradient Algorithm in CR $1 Y$ superromputers. Technical Report. Lawrence Livermore National Lab. Livermore. ('A, 19ki.

[16] 1. Lichnewhi. Som recror and parallel implementations for preconditioned gratient algorithms. In J. Kowalik, editor. Procedings of the VATO workshop on high sperd computing. pages 343-359. 1984.

[1i] T. A. Manteuffel. The Shifted Incomplete Cholesky Factorization. Technical Report S.ANDT-8226. Sandia I aboratories, May 1978.

[18] J. A. Weijerink and H. A. Van der Vorst. An iterative solution method for linear equation systems of which the coefficient matrix is a symmetric .I-Matrix. Wath. Comp. 31:1 19 162, 1977.

[19] R. G. Welhem. Solıtion of linear svstems with striped sparse matrices. Parallel Computing. 6:165 184, 1988.

[20] G Meurant. Multitasking the conjugate gradient method on the CR.AY XYIP/1R. Parallel Computing. $5: 267-280,198 i$.

[21] 1. Osterhallg. Cinide to Parallal Programming on Siquent Computer Systenas. Sequent Computer Systems, Inc., 1986. Sequent Technical Publications.

[22] C. V. Ramamoorthy, K. . . Chandry and W. J. Gonzales Jr. Optimal scheduling strategies in a multiprocessor system. IEEE Trans. Comput., c-21(2):1:3i$1 \downarrow 6$, Feb 1972 . 
[23] J. K. Reid. On the method of conjugate gradients for the solution of large sparse systems of linear equations. In J. K. Reid, editor, Large Sparse Sets of Linear Equations, pages 231-254, Academic Press. New York, 1971.

[24] Y. Saad. Krylov Subspace lethods on Supercomputers. Technical Report TR88.40, RIACS, NASA .imes Research Center. Moffett Field, C.194035, September 1988. To appear SIAM J. SCI. STAT COMPTT.

[25] J. Saltz. R. Mirchandaney, and D. Baxter. Run-Time Parallelization and Scheduling of Loops. Technical Report ICASE Report No. 83-70, IC ASE. N.ASA Langley Research Center, Hampton. VA 23665, December 1982.

[26] M. K. Seager. Parallelizing Conjugate Gradient Mfthod for the C'R.AY X-MP. Technical Report, Lawrence Livermore National Lab, Livermore. CA. 1984.

[27] Omar Wing and John W. Huang. I computational model of parallel solution of linear equations. IEEE Trans, on Computers, 29( 7 ):632-638, 1980. 


\section{A Test Problems}

For this work we have chosen 7 test cases which are representative of the class of problems solved by iterative mothods. All are from two-dimensional domains. The first five are from the Harwell-Boeing collection [ $B$ ] and the last two are from electro-magnetic analysis [:]. The test cases are described in Table 8.

\begin{tabular}{|c|c|c|c|c|}
\hline Case & Ref. & Description & Order & Vonzeros \\
\hline 1 & {$[Q]$} & $\begin{array}{l}\text { I nine point discretization of the Laplacian on } \\
\text { a nuit iquare with Dirichlet boundary condi- } \\
\text { tions. LAP30 }\end{array}$ & 900 & 4322 \\
\hline 2 & {$[x]$} & $\begin{array}{l}\text { Matrix ned in modeling power system net- } \\
\text { works. PS.tDMITI }\end{array}$ & 662 & $156 x$ \\
\hline 3 & {$[y]$} & $\begin{array}{l}\text { Matrix ned in modeling power system net- } \\
\text { works. PSADMIIT2 }\end{array}$ & 191 & 1080 \\
\hline$t$ & {$[x]$} & $\begin{array}{l}\text { Matrix used in modeling power system net- } \\
\text { works. PS.DDMIT3 }\end{array}$ & 68.5 & 1967 \\
\hline$;$ & {$[x]$} & $\begin{array}{l}\text { Matrix used in modeling power system net- } \\
\text { works. PSADMITt }\end{array}$ & 1138 & 2596 \\
\hline 6 & {$[3]$} & $\begin{array}{l}\text { A firstorder triangular finite element dis- } \\
\text { cretization of the Laplacian operator on a unit } \\
\text { square. }\end{array}$ & 2500 & 7251 \\
\hline 7 & {$[i]$} & $\begin{array}{l}\text { Matrix from a nonlinear magnetostatic } \\
\text { model of a pormanent magnet motor, us- } \\
\text { ing an unstructured finite plement mesh with } \\
\text { mixed triangular and quadrilateral third- } \\
\text { order alements. }\end{array}$ & 6.517 & $69.6 \div 0$ \\
\hline
\end{tabular}

Table R: Test Case Descriptions 


\section{B Sequent Overview and Performance Figures}

This section provides an orerview of the architecture of the Sequent Balance 21000 and the execution times for the operations that wore used in the rimings given in this paper. The architectural description is due to Osterhaug [21].

\section{B.1 The Sequent Architecture}

The Sequent Balance 21000 is a shared memory multiprocessor. The processors are identical $10-\mathrm{MHz}$ National Semicondactor 32032 s. These are 32-bit processors. They operate on a peer basis, executing a single copy of the operating sistems executive, or "kernel".

There is no designated "master" cpu. All processors. memory modıles, and i/o controllers plig into a single high-speed bus. There is hardware support for mutual exchusion - to support exclusive access to shared data structures, the system includes $u p$ to $6 \mathrm{th}$ user-accessible hardware spin-locks.

The system we used has 12 processors and 28 Mbytes of inemory. In addition. each cpu has 8 Kbytes of local R.AM and 8 Kbytes of cache R.IM. The local RAM holds a copy of certain frequently used kernel code and read-only kernel data structures. The cache RAM holds blocks of system memory most recently used by the cpu.

\begin{tabular}{||l|c|c|c||}
\hline \multirow{2}{*}{ Operation } & \multicolumn{3}{|c||}{ Operand } \\
\cline { 2 - 4 } & 4-Byte Integer & t-Bye Real & 3-Byte Real \\
\hline Addition & 4.4 & 32.4 & 18.9 \\
Multiplication & 12.7 & 28.1 & 20.8 \\
Division & 17.0 & 33.0 & 25.5 \\
\hline
\end{tabular}

Table 9: Time in $\mu$ seconds for Arithmetic Operations

\section{B.2 System Timing}

This section provides execution times in microseconds for a variely of operations that were used by the programs discussed in this paper. Times for arithuntic operations are shown in Table 9. These timings are computed by looping through a program segment 50.000 times. The time before the loop was executed was then subracted from the time at the end of the loop. Some time was subtracted for loop orerhead and then that time was divided by the number of iterations throngh the loop. 
Locking and unlocking of locations in the hardware atomic lock memory was done by the in-line (' macros SLOCK() and S_UNLOCK(). If we assinme there is no contention for the lork. locking and unlocking a lock takes a total of 3.5 microseconds. The system provided routines in the Paralle] Programming Library were slower, taking 53 microseconds. I function. m_next(). is provided to increment a global counter and return the current value. This function takes an average of 19 microseconds per call. 


\section{Memory Access Times on the Sequent}

In the IC'CG method every element of $L, L^{T}$, and $t$ are read once each iteration. To explain the inefficiency of Test Case $i$ we ran a simple tust that itrates over different array sizes accessing each element once per iteration. We measured the average time to access an array element as a function of the size of the array. We created a program with a double-precision array, big-array[]. with 200.000 elements. Then, we timed the following two loops:

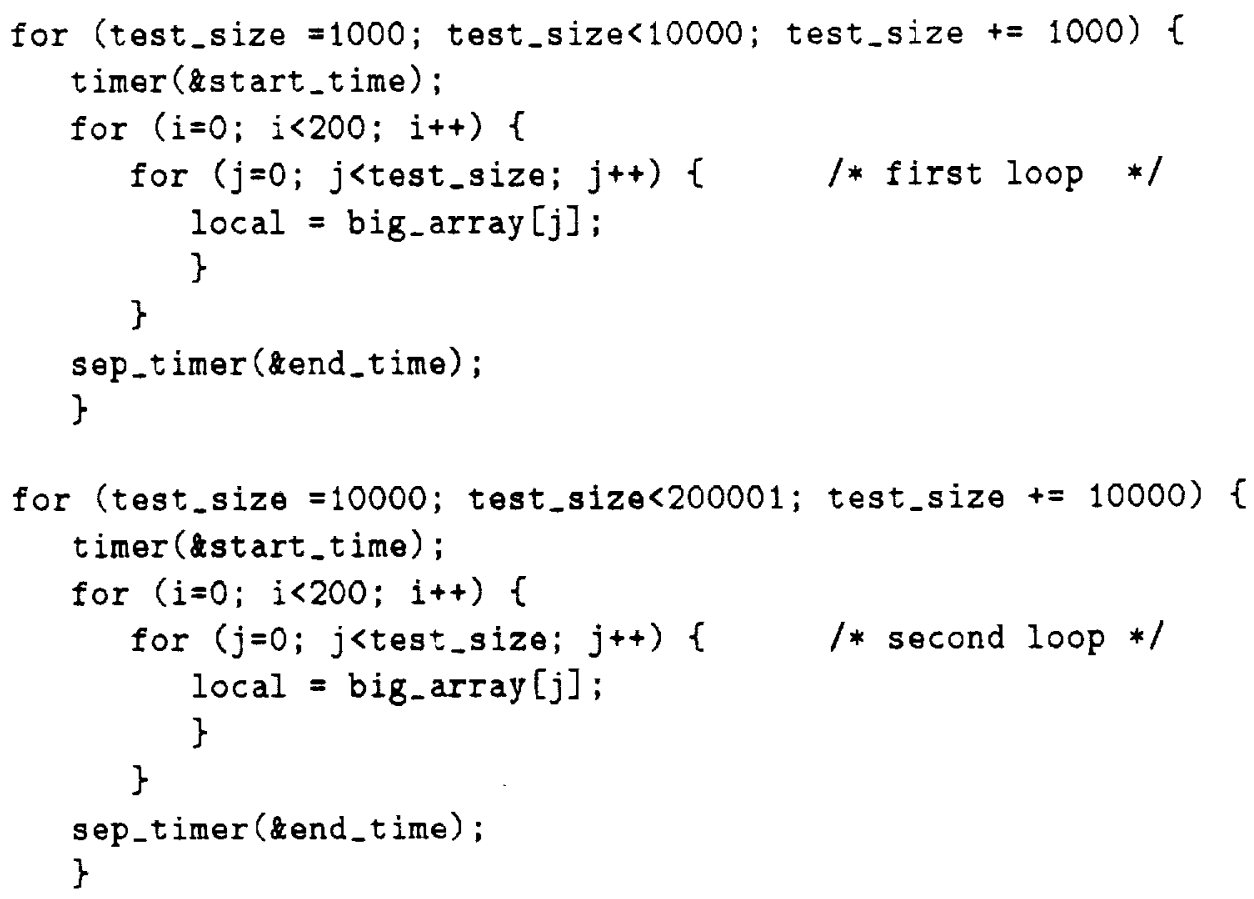

We copy the elements of the array one at a time to a scalar variable local. In the first loop, the number of array elements accessed, test_size. varies from 1.000 to 9.000 in increments of 1,000 . In the second loop, the number of array elements accessed. test_size, varies from 10,000 to 100.000 in increments of 10.000 . We loop 200 times for each test size and divide the time by the total nrmber of array accesses. This was done 5 times for each test and the results wre areraged.

The timing routine returns both user time (utime) and sistem time (stime) separately rather than the sum of the two Wo used the sum in all previous timings. These quantities are defined as follows:

user time the total amount of time spent executing in user mode 


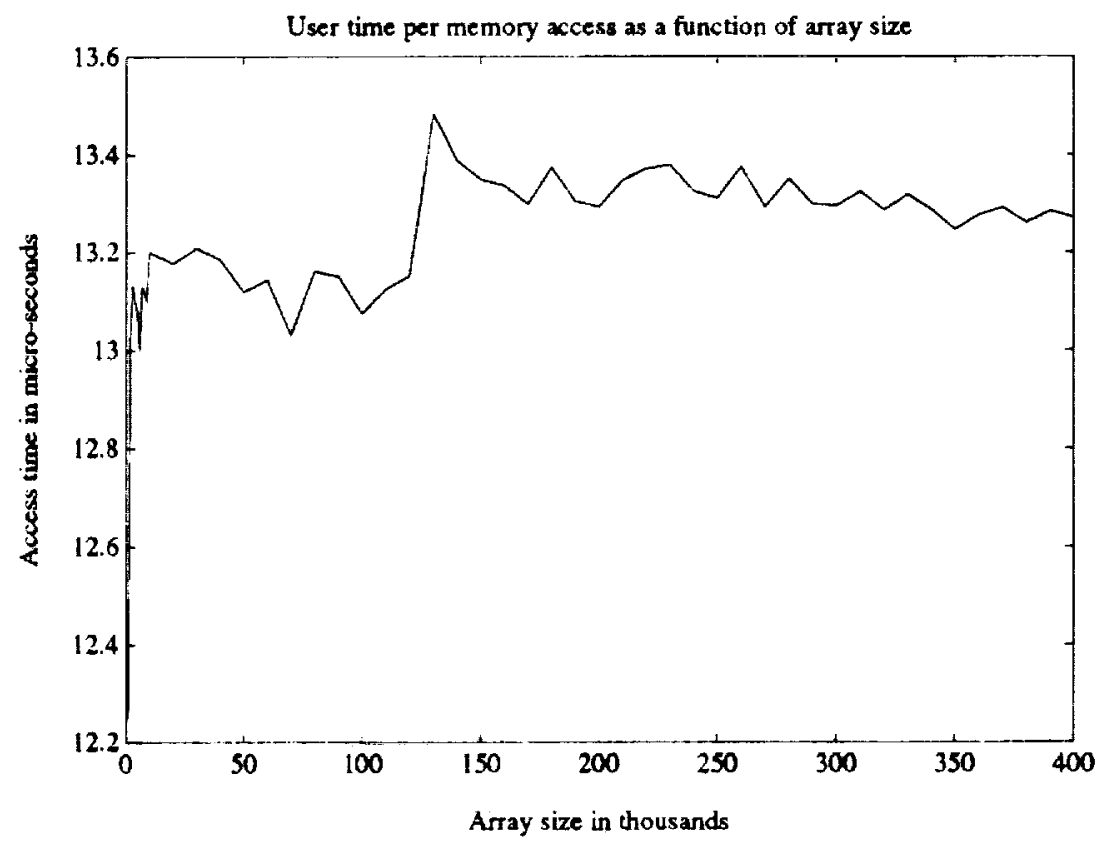

Fignre $i$ : Ter Time in $\mu$ secs for Menory tccess as a function of Array Size

system time the total amount of time spent in the system on behalf of the process.

The results of this test are shown in Figures 7 and 8 . The times are measured in microseconds. There is approximately a $2 \%$ increase in user time per access as the array size is increased from $120 \mathrm{~K}$ to $130 \mathrm{~K}$. But, there is a factor of $\bar{j}$ increase in system time per access as the number of array elements in the test case increases from $120 \mathrm{~K}$ to $130 \mathrm{~K}$. Recall, the total storage for full $A$ in Test Case $T$ is $132 . \times 23$ double-precision numbers. The Sequent takes longer to access each element for this large problem than for all the other test cases.

In Table 10 we show separate entries for the user time and the ststem time for the sequential. symmetric and full matrix implementations of Test Case 7 . The feature to notice is the drastic increase in system tim* for the forward and backward iolve in the "Full" care as compared with the corresponding times of the "Symmetric" and the "Sequential" implomentations. This is partially explained by the test loops above. We see that large problems such as Tust (ase 7 are not efficient on the Sequent. 


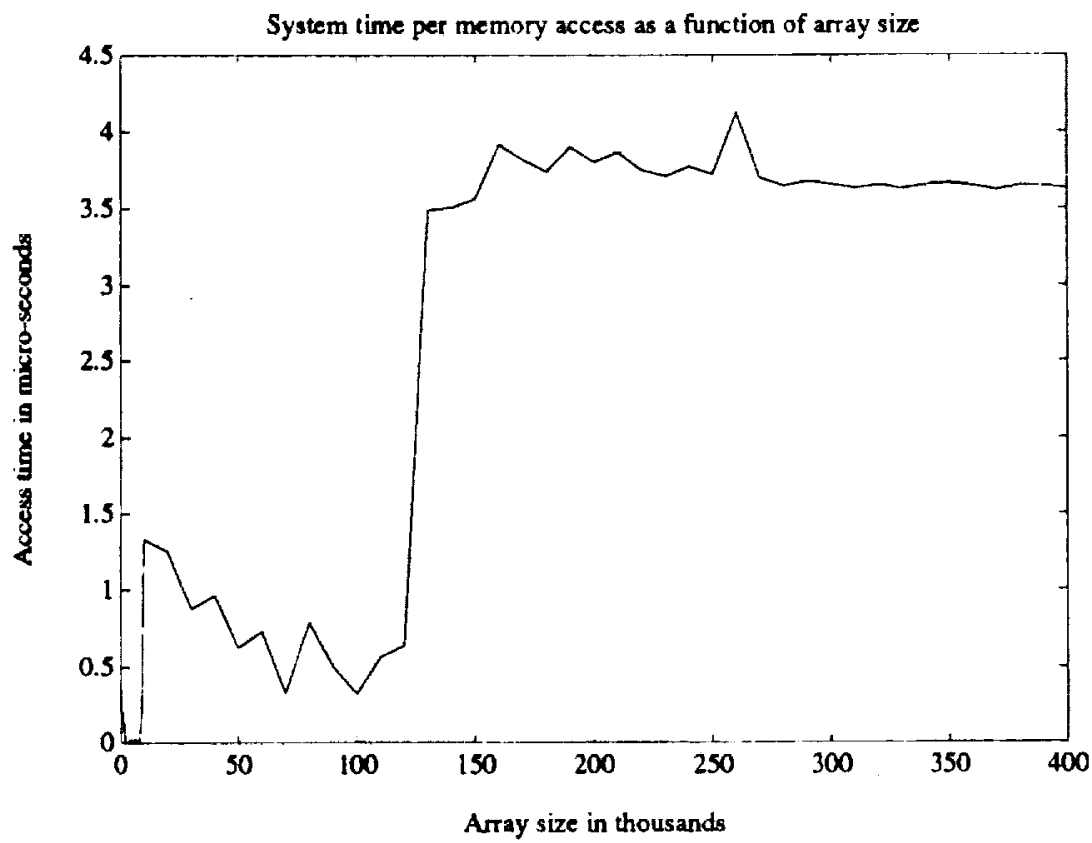

Figure $\$$ : System Time in $\mu$ secs for Memory Access as a function of Array Size

\begin{tabular}{|c|c|c|c|c|c|}
\hline \multirow[t]{2}{*}{ Problem } & & \multicolumn{4}{|c|}{ Operation } \\
\hline & & fwd & bck & mult & total \\
\hline \multirow{3}{*}{ sequential } & utime & 576.05 & 506.50 & 11.57 .10 & 2535.25 \\
\hline & stime & 94.53 & $4.6 \vec{\imath}$ & 119.74 & 2.54 .36 \\
\hline & totals & 670.58 & 311.17 & 1277.14 & 2829.61 \\
\hline \multirow{3}{*}{$\begin{array}{l}\text { symmetric } \\
\text { (parallel) }\end{array}$} & utime & 117.97 & 65.85 & 182.06 & $39 \overline{7} .5 T$ \\
\hline & stime & 88.84 & 19.95 & $143.9 \vec{\imath}$ & 257.82 \\
\hline & totals & 206.81 & 8.5 .80 & 326.03 & 655.39 \\
\hline \multirow{3}{*}{$\begin{array}{l}\text { full } \\
\text { (parallel) }\end{array}$} & utime & 73.33 & 72.17 & 173.28 & 349.94 \\
\hline & stime & 127.19 & 144.46 & 34.18 & 310.25 \\
\hline & totals & 200.52 & 216.63 & $20 \bar{\pi} .46$ & 660.19 \\
\hline
\end{tabular}

Table 10: Detailed timing of Case $i$ in seconds 


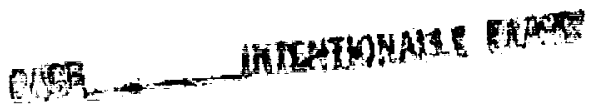

